Research Paper

\title{
Acid-electrolyzed functional water induces extracellular matrix metalloproteinase inducer, a possible novel alarmin, secretion from oral squamous cell carcinoma cell lines
}

\author{
Masafumi Kusunoki ${ }^{1}$, Eri Sata ${ }^{2,3}$, Kensuke Nishio ${ }^{4}$, Takayoshi Tanaka ${ }^{5,6}$, Tetsuya Nishida ${ }^{1,7}$, Naoyuki \\ Sugano $^{1,7}$, Shuichi Sato ${ }^{1,7}$, Masatake Asano ${ }^{8,9, 凹}$ \\ 1. Department of Periodontology, Nihon University School of Dentistry, 1-8-13 Kanda Surugadai, Chiyoda-ku, Tokyo 101-8310, Japan \\ 2. Department of Orthodontics, Nihon University School of Dentistry, 1-8-13 Kanda Surugadai, Chiyoda-ku, Tokyo 101-8310, Japan \\ 3. Oral Structural and Functional Biology, Nihon University Graduate School of Dentistry, 1-8-13 Kanda Surugadai, Chiyoda-ku, Tokyo 101-8310, Japan \\ 4. Department of Complete Denture Prosthodontics, Nihon University School of Dentistry, 1-8-13 Kanda Surugadai, Chiyoda-ku, Tokyo 101-8310, Japan \\ 5. Maxillofacial Prosthetic Clinic, Nihon University School of Dentistry, Tokyo, Japan \\ 6. Department of Oral and Maxillofacial Surgery, Nihon University School of Dentistry, 1-8-13 Kanda Surugadai, Chiyoda-ku, Tokyo 101-8310, Japan \\ 7. Division of Advanced Dental Treatment, Dental Research Center, Nihon University School of Dentistry, 1-8-13 Kanda Surugadai, Chiyoda-ku, Tokyo \\ 101-8310, Japan \\ 8. Department of Pathology, Nihon University School of Dentistry, 1-8-13 Kanda Surugadai, Chiyoda-ku, Tokyo 101-8310, Japan \\ 9. Division of Immunology and Pathobiology, Nihon University School of Dentistry, 1-8-13 Kanda Surugadai, Chiyoda-ku, Tokyo 101-8310, Japan
}

$\triangle$ Corresponding author: Department of Pathology, Nihon University, School of Dentistry, 1-8-13 Kanda Surugadai, Chiyoda-ku, Tokyo 101-8310, Japan. +81-3-3219-8114; asano.masatake@nihon-u.ac.jp

(C) Ivyspring International Publisher. This is an open access article distributed under the terms of the Creative Commons Attribution (CC BY-NC) license (https://creativecommons.org/licenses/by-nc/4.0/). See http://ivyspring.com/terms for full terms and conditions.

Received: 2018.03.20; Accepted: 2018.06.30; Published: 2018.09.07

\begin{abstract}
Extracellular matrix metalloproteinase inducer (EMMPRIN) secretion was induced in the oral squamous cell carcinoma cell line HSC3 cell by acid-electrolyzed functional water (FW) stimulation. Augmented EMMPRIN secretion was not under transcriptional control; rather, it was derived from the intracellular storages. EMMPRIN secretion was also induced under oxidative stress and accompanied by the release of lactate dehydrogenase (LDH). The molecules released from cells undergoing necrosis are called as alarmins, and the secretion of IL-1 $\alpha$, a typical alarmin, was induced by FW stimulation and oxidative stress. Intracellular localization was examined by cell fractionation. A significant amount of EMMPRIN was localized in the triton X-100 and DNase sensitive fractions; the levels were drastically reduced following FW treatment. The function of the released EMMPRIN was examined using the monocytic cell line THPI. Culture supernatant derived from FW-treated HSC3 cells induced the expression of matrix metalloproteinases (MMPs) 1, 2, 8, 9, 13, and 14, platelet-derived growth factor, and interleukin-8. In contrast, vascular endothelial growth factor expression was reduced. Induction of these factors was abolished following eliminating of EMMPRIN by immunoprecipitation. These results indicate that EMMPRIN might be considered as a type of alarmin that transduces danger signals to the surrounding cells.
\end{abstract}

Key words: Acid-electrolyzed functional water, Extracellular matrix metalloproteinase inducer, Oral squamous cell carcinoma, Matrix metalloproteinase

\section{Introduction}

Cancer cells degrade the surrounding tissue by producing enzymes such as matrix metalloproteinases (MMPs), thus enabling tumor invasion [1]. Initially, MMPs were thought to be produced mainly by the tumor cells; however, histological examinations of the pattern of MMP expression in cancer specimens revealed that most of them are produced by stromal fibroblasts [2]. Tumor 
cells can stimulate the surrounding fibroblasts to produce higher levels of MMPs within the tumor microenvironment via the action of the extracellular matrix metalloproteinase inducer (EMMPRIN), which belongs to the immunoglobulin superfamily [3]. EMMPRIN (renamed as CD147) is a transmembrane $28 \mathrm{kDa}$ glycoprotein composed of an extracellular domain of 187 amino acid residues (aa), a transmembrane domain (24 aa) and a cytoplasmic domains (40 aa) [4-6]. The extracellular region contains two immunoglobulin domains and three $\mathrm{N}$-glycosylation sites [7]. Depending on the degree of glycosylation, it migrates to various positions on the SDS-PAGE [6]. The glycosylation status of this protein is important for its biological functions [8]. The charged amino acid residue (glutamic acid) localized in the transmembrane region allows for the association of EMMPRIN with other transmembrane proteins [7]. Besides its role in cancer progression, EMMPRIN plays pleiotropic roles in development, reproduction, and wound healing [3].

Electrolytically-generated acid functional water (FW), obtained by electrolyzing low concentrations of saline, is widely used as a disinfectant, in clinical practice [9]. In a previous report, FW was demonstrated to augment basic fibroblast growth factor (bFGF) secretion in HeLa cells [10]. As FW-treatment significantly induced the secretion of lactate dehydrogenase (LDH), a marker of cell damage, it was expected to cause some amount of damage to the cells. The molecules released from the damaged cells are referred to as alarmins [11]. In the present study, we attempted to examine the effect of FW on oral squamous cell carcinoma-derived cell lines. FW-treatment was found to induce the secretion of EMMPRIN; therefore, the aim of the study was to present EMMPRIN as a novel alarmin.

\section{Materials and Methods}

\section{Reagents}

The FW (actual chloride concentration (ACC) 30 ppm, pH 2.7, oxidation-reduction potential (ORP) of more than $1100 \mathrm{mV}$ ) was kindly provided by Miura Denshi (Akita, Japan). Anti-human CD 147 antibody $(\mathrm{Ab})$ (anti-EMMPRIN $\mathrm{Ab}$ ) was purchased from BioLegend (San Diego, USA).

\section{Cell culture and FW stimulation}

Human oral squamous cell carcinoma cell lines HSC3 and Ca9-22, and the human monocytic cell line THP1 were obtained from the Health Science Research Resources Bank (Osaka, Japan). The cells were maintained in RPMI1640 medium supplemented with $10 \% \mathrm{FCS}, 50 \mathrm{mg} / \mathrm{ml}$ streptomycin, and $50 \mathrm{U} / \mathrm{ml}$ penicillin (10\% FCS-RPMI). The HSC3 cells were plated on a 24-well plate (IWAKI, Tokyo, Japan) at a density of $1 \times 10^{5} /$ well. The cells were washed with PBS and treated with FW for $30 \mathrm{~s}$. The stimulation was stopped by adding 10\% FCS-RPMI and further cultured for the indicated time periods.

\section{Cytokine Array Experiment}

HSC 3 cells were plated on a $10 \mathrm{~cm}$ cell culture dish (Greiner, Tokyo, Japan) at a density of $1 \times 10^{6}$ /dish on the day before the experiment. Following stimulation with FW for $30 \mathrm{~s}$, the cells were further cultured for $18 \mathrm{~h}$. The culture supernatants were collected and subjected to cytokine array experiments using the Human XL Cytokine Array kit (R\&D systems) according to the manufacture's protocol. Images were taken using ChemiDoc XRS (BioRad, Tokyo, Japan).

\section{Enzyme-Linked Immunosorbent Assay (ELISA)}

The cells were cultured for 1, 3, 6 and $12 \mathrm{~h}$ and the culture supernatants were harvested to measure the concentration of EMMPRIN. The cells were washed with PBS and lysed with cell lysis buffer (50 $\mathrm{mM}$ Tris- $\mathrm{HCl}, \mathrm{pH} 7.5,150 \mathrm{mM} \mathrm{NaCl}$, and $0.5 \%$ TritonX-100). EMMPRIN concentrations were measured using the DuoSet ELISA Development System (R\&D Systems).

\section{Real-time PCR}

Total RNA was purified using the RNeasy mini kit (QIAGEN, Tokyo, Japan). cDNA was synthesized using Superscript III reverse transcriptase (Invitrogen, San Diego, CA, USA) and subjected to real-time PCR, as described previously [12]. Real-time PCR was performed using the CFX96-Real-Time-System (BioRad, Tokyo, Japan) with SYBR green (TaKaRa, Tokyo, Japan). The primers used in this study are listed in Table 1.

\section{Cell stimulation and LDH measurement}

Lactate dehydrogenase (LDH) was measured by treating the cells with either $\mathrm{FW}$ or $10 \mathrm{mM} \mathrm{H}_{2} \mathrm{O}_{2}$, or culturing them at $42^{\circ} \mathrm{C}$. After treatment, the cells were further cultured for $3 \mathrm{~h}$. The culture supernatants were harvested and subjected to LDH measurement using an LDH activity assay kit (Sigma-Aldrich, Tokyo, Japan). The samples were also subjected to EMMPRIN and IL-1a concentration measurements using the DuoSet ELISA Development System (R\&D Systems). 
Table 1. Primers used in this study

\begin{tabular}{lll}
\hline Gene & Forward primer & Reverse primer \\
\hline$\beta$-actin & GGA GCA AGT ATC TTG ATC TTC & GGA GCA AGT ATC TTG ATC TTC \\
MMP1 & GGG GCT TTG ATG TAC CCT AGC & TGT CAC ACG CTT TTG GGG TTT \\
MMP2 & CCC $\Lambda$ CT GCG GTT TTC TCG $\Lambda$ T & C $\Lambda$ $\Lambda$ GG GGT $\Lambda$ TC CAT CGC CAT \\
MMP8 & TGC TCT TAC TCC ATG TGC ACA & TCC AGG TAG TCC TGA ACA GTT \\
MMP9 & GGG ACG CAG ACA TCG TCA & TCG TCA TCC TCG AAA TGG \\
MMP13 & TCC TGA TGI GGG TGA ATA CAA & GCC ATC GIG AAG TCT GGI AAA \\
MMP14 & CGA GGT GCC CTA TGC CTA & CTC GGC AGA GTC AAA GTG \\
IL-8 & TT'T TGC CAA GGA GTG CTA AAG & AAC CCT C IG CAC CCA GTT TTC \\
VEGF & AGG GCA GAA TCA TCA CGA AGT & AGG GTC TCG ATT GGA TGG CA \\
PDGF & AGC ACC TTC GTT CTG ACC TG & TAT TCT CCC GTG TCT AGC CCA \\
EMMPRIN & GCA GCG GGC AGC ACC & CCA CCT GCC TCA GGA AGA GTT \\
\hline
\end{tabular}

\section{Cell fractionation experiment}

HSC 3 cells were plated on a $15 \mathrm{~cm}$ dish $(1 \times$ $10^{7} /$ dish). The cells were washed twice with PBS, and stimulated with or without FW for $30 \mathrm{~s}$ and further cultured for $1 \mathrm{~h}$. The cells were collected, washed with PBS and subjected to cell fractionation as described previously [13]. Briefly, the cells were re-suspended with Triton X-100 buffer $(0.1 \%$ Triton X-100, $10 \mathrm{mM}$ HEPES, pH7.3, $100 \mathrm{mM} \mathrm{NaCl}, 0.1 \mathrm{mM} \mathrm{MgCl} 2,0.1 \mathrm{mM}$ PMSF, $1.0 \mathrm{mM}$ DTT and proteinase inhibitor) and incubated on ice for $10 \mathrm{~min}$. The cells were pelleted by centrifugation at $1,000 \times \mathrm{g}$ at $4^{\circ} \mathrm{C}$ for $5 \mathrm{~min}$. The supernatant was collected as the " 1 st fraction". Next, the cell pellets were re-suspended with Triton X-100 buffer and pelleted by centrifugation at 1,000 $\times \mathrm{g}$ for 5 min. The supernatant was collected as the "Wash fraction". The cell pellets were re-suspended with Triton X-100 buffer containing $1000 \mathrm{U} / \mathrm{ml}$ DNase and incubated at $25^{\circ} \mathrm{C}$ for $1 \mathrm{~h}$. Then, the cells were pelleted by centrifugation at $1,000 \times \mathrm{g}$ for $5 \mathrm{~min}$. The supernatant was collected as the "DNase fraction". The cell pellets were re-suspended with $2 \mathrm{M} \mathrm{NaCl}$ (0.1\% Triton X-100, 10 mM HEPES, pH7.3, 2M NaCl, $0.1 \mathrm{mM} \mathrm{MgCl} 2,0.1 \mathrm{mM}$ PMSF, $1.0 \mathrm{mM}$ DTT and inhibitor) and incubated on ice for $10 \mathrm{~min}$. The cells were pelleted by centrifugation at 1,000 $\times \mathrm{g}$ for $5 \mathrm{~min}$ and the supernatant was collected as the "NaCl fraction". Finally, the cell pellets were re-suspended with 1\% SDS (10 mM HEPEs, pH7.3, $100 \mathrm{mM} \mathrm{NaCl}$, $0.1 \mathrm{mM} \mathrm{MgCl}_{2}, 0.1 \mathrm{mM}$ PMSF, $1.0 \mathrm{mM}$ DTT, 1\% SDS and inhibitor) and incubated at room temperature for $10 \mathrm{~min}$. Then, the cells were pelleted by centrifugation at $15,000 \times \mathrm{g}$ at $25^{\circ} \mathrm{C}$ for $5 \mathrm{~min}$ and the supernatants were collected as "SDS fraction". The protein concentrations of each fraction were measured using the Bio Rad protein assay kit (BioRad, Tokyo, Japan) and subjected to Western blotting.

\section{THPI stimulation}

The conditioned medium derived from FW-stimulated or un-stimulated HSC3 cells were collected for THP1 cell stimulation. The THP1 cells were cultured with or without the conditioned medium for $24 \mathrm{~h}$, harvested and total RNA was extracted. cDNA was generated and real-time PCR was performed as described earlier. For the inhibition experiments, the EMMPRIN in the conditioned medium was immunoprecipitated with anti-EMMPRIN antibody followed by protein G-sepharose (HE Healthcare, Tokyo, Japan). The samples were collected after centrifugation $(10,000 \times$ $\mathrm{g}, 3 \mathrm{~min}$ ) and subjected to THP1 stimulation.

\section{Statistical analysis}

Student's t-test or two-way ANOVA with Tukey tests were used for the statistical analysis. Results are presented as mean \pm standard deviation (SD). P values $<0.05$ were considered as statistically significant.

\section{Results}

The cytokine secretion profiles were compared between the PBS- and FW-treated samples (Fig 1). The expression levels of most of the cytokines secreted in the PBS-treated samples were decreased following FW-treatment. On the other hand, the levels of EMMPRIN, IL-1a, IL-1ra and IL-32 were significantly augmented in the FW-treated samples (Fig 1, lower panel). Among them, EMMPRIN level was most prominently increased; hence, we focused on this molecule in the following experiments. 


\begin{tabular}{|c|c|c|c|c|c|c|c|c|c|c|c|c|}
\hline & 12 & 34 & 56 & 78 & 10 & 1112 & $13 \quad 14$ & $15 \quad 16$ & 1718 & 1920 & 21 & $23 \quad 24$ \\
\hline $\mathrm{A}$ & controll(+) & Acrp30 & ApoA-1 & Angogenin & ANGPT1 & ANGPT2 & BAFF & BDNF & C5a & CD14 & CD30 & control(+) \\
\hline B & & CD4OL & CHI3L1 & CFD & CRP & Cripto-1 & CystaitinC & Dkk-1 & DPPIV & EGF & Emmprin & \\
\hline$c$ & & ENA.78 & Endoglin & FasLigand & FGF-2 & KGF & FGF-19 & FLT3LG & G.CSF & GDF-15 & GM-CSF & \\
\hline D & GROa & GH & HGF & ICAM-1 & IFN-Y & IGFBP.2 & IGFBP.3 & IL-1ब & |L-1及 & |L-Ara & IL-2 & |L-3 \\
\hline E & |L.-4 & IL.5 & IL-6 & IL-8 & IL-10 & IL-11 & IL-12p70 & |L-13 & |L-15 & |L-16 & IL-17A & |L-18Bpa \\
\hline $\mathrm{F}$ & IL-19 & IL-22 & IL-23 & IL-24 & IL-27 & IL-31 & IL-32 & IL-33 & |L-34 & PP.10 & I-TAC & KLK3 \\
\hline$G$ & Leptin & LIF & LCN2 & MCP-1 & MCP-3 & M-CSF & MIF & MIG & MIP.1a & MIP. $3 a$ & MIP. $3 \beta$ & MMP-9 \\
\hline H & MPO & OPN & PDGF-AA & PDGF-AB & Pentraxin3 & PF4 & RAGE & RANTES & RBP-4 & Relaxin-2 & Resistin & SDF-1a \\
\hline I & SerpinE1 & SHBG & ST2 & TARC & TFF3 & TfR & TGF- $\alpha$ & THBS1 & TNF-a & UPAR & VEGF & \\
\hline J & control( + ) & & VDB & CD31 & TIM-3 & VCAM-1 & & & & & & control(-) \\
\hline
\end{tabular}

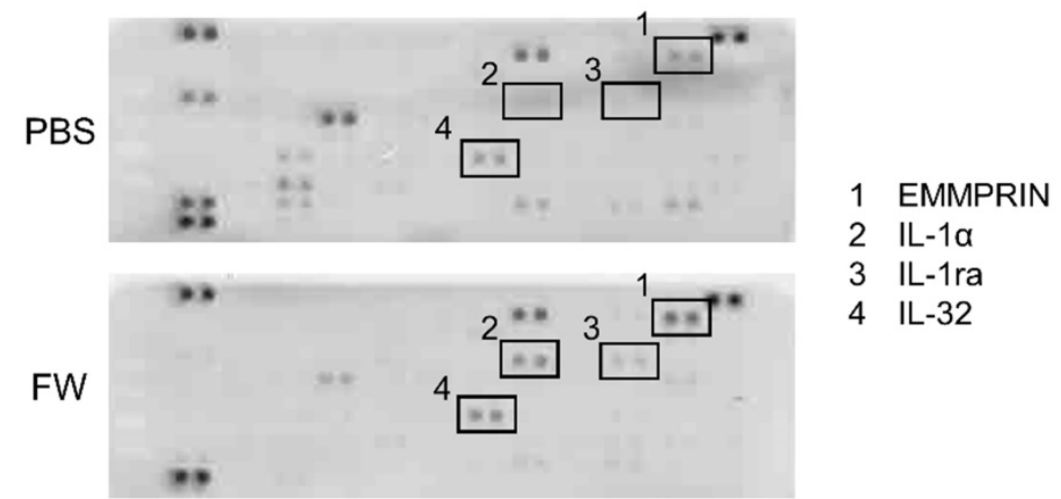

Figure 1. Electrolytically-generated acid functional water (FW) treatment significantly augmented EMMPRIN secretion. HSC3 cells were treated with PBS (upper panel) or FW (lower panel) for $30 \mathrm{~s}$. The treatment was stopped by adding 10\% FCS-RPMI and the cells were washed. After wash, the cells were further cultured for $18 \mathrm{~h}$, the supernatants were harvested and subjected to a cytokine array experiment. The positions of the cytokines were spotted on the membrane. The dots in rectangles showed 1 . EMMPRIN, 2. IL-1 1 , 3. IL-1 receptor antagonist (IL-1 ra) and 4. IL-32, respectively. All these factors were augmented by FW-treatment. The representatives of two independent experiments are shown.

To confirm the increase in EMMPRIN levels, HSC 3 and Ca9-22 cells were treated with FW, and the culture supernatants were harvested and subjected to ELISA (Fig 2). EMMPRIN levels had increased to 1700 $\mathrm{pg} / \mathrm{ml}$ in the HSC3 cells and $1620 \mathrm{pg} / \mathrm{ml}$ in the Ca9-22 cells after $1 \mathrm{~h}$ of culture (Fig 2A and C). The levels were further increased to 2400 (HSC3) and 2450 $\mathrm{pg} / \mathrm{ml}(\mathrm{Ca} 9-22)$ at $12 \mathrm{~h}$ (Fig 2A and C). In PBS-treated samples, EMMPRIN concentration was less than 500 $\mathrm{pg} / \mathrm{ml}$ in both cell lines, whereas, in the cell lysates EMMPRIN concentrations had decreased drastically, in a culture time-dependent manner, following FW-treatment (Fig 2 B, D).

To examine whether augmented EMMPRIN secretion was regulated at the transcriptional level, total RNA was purified after $0.5,1,3,6$, and $12 \mathrm{~h}$ of stimulation and subjected to real-time PCR. EMMPRIN mRNA levels were not drastically altered during the indicated time points in either of the cell lines (Fig S1). FW-treatment was expected to exert a certain amount of stress on the cells, and the induction of EMMPRIN might have been the result of this stress-related response. To examine this possibility, HSC3 cells were cultured in the presence of $10 \mathrm{mM}$ $\mathrm{H}_{2} \mathrm{O}_{2}$ or at $42^{\circ} \mathrm{C}$ for $3 \mathrm{~h}$ and the concentrations of secreted EMMPRIN were measured (Fig 3). Significant EMMPRIN secretion was observed with FW $(1,610 \mathrm{pg} / \mathrm{ml})$ and $1 \% \mathrm{H}_{2} \mathrm{O}_{2}(1,150 \mathrm{pg} / \mathrm{ml})$, whereas culturing at $42^{\circ} \mathrm{C}$ induced a slight increase in
EMMPRIN secretion $(400 \mathrm{pg} / \mathrm{ml})$, statistical significance notwithstanding (Fig 3A). The extent of cell damage was monitored by measuring $\mathrm{LDH}$ concentrations (Fig 3B). The most prominent LDH release was observed after FW-treatment (127 nkat $/ \mathrm{ml}$ ) followed by the $1 \% \mathrm{H}_{2} \mathrm{O}_{2}$ (123 nkat/ml) treatment, and to a lesser extent after the $42^{\circ} \mathrm{C}$ culture (49 nkat/ml; Fig. 3B). The molecules released from the stressed cells are called as alarmins. To examine the candidature of EMMPRIN as an alarmin, the release of the representative alarmin cytokine IL-1a was measured (Fig 3C). IL-1a secretion was significantly augmented after $\mathrm{FW}$ and $1 \% \mathrm{H}_{2} \mathrm{O}_{2}$ treatment, but only slightly after culturing at $42^{\circ} \mathrm{C}$ (Fig 3C).

Intracellular localization of EMMPRIN in HSC3 cells was examined by the cell fractionation assay. The FW-treated or un-treated HSC3 cells were sequentially processed with Triton X-100 based buffer containing various reagents. Each fraction was subjected to Western blotting. The main $66 \mathrm{kDa}$ band was detected in the 1 st, wash and DNase fractions (Fig 4, upper panel). The same fractionation experiment was performed after FW treatment and compared with the control. The $66 \mathrm{kDa}$ band was detected in the same fractions as in the control; however, the intensities were significantly lower in FW-treated sample (Fig 4, lower panel). These findings indicated that FW treatment drastically reduced EMMPRIN levels in the cells. 

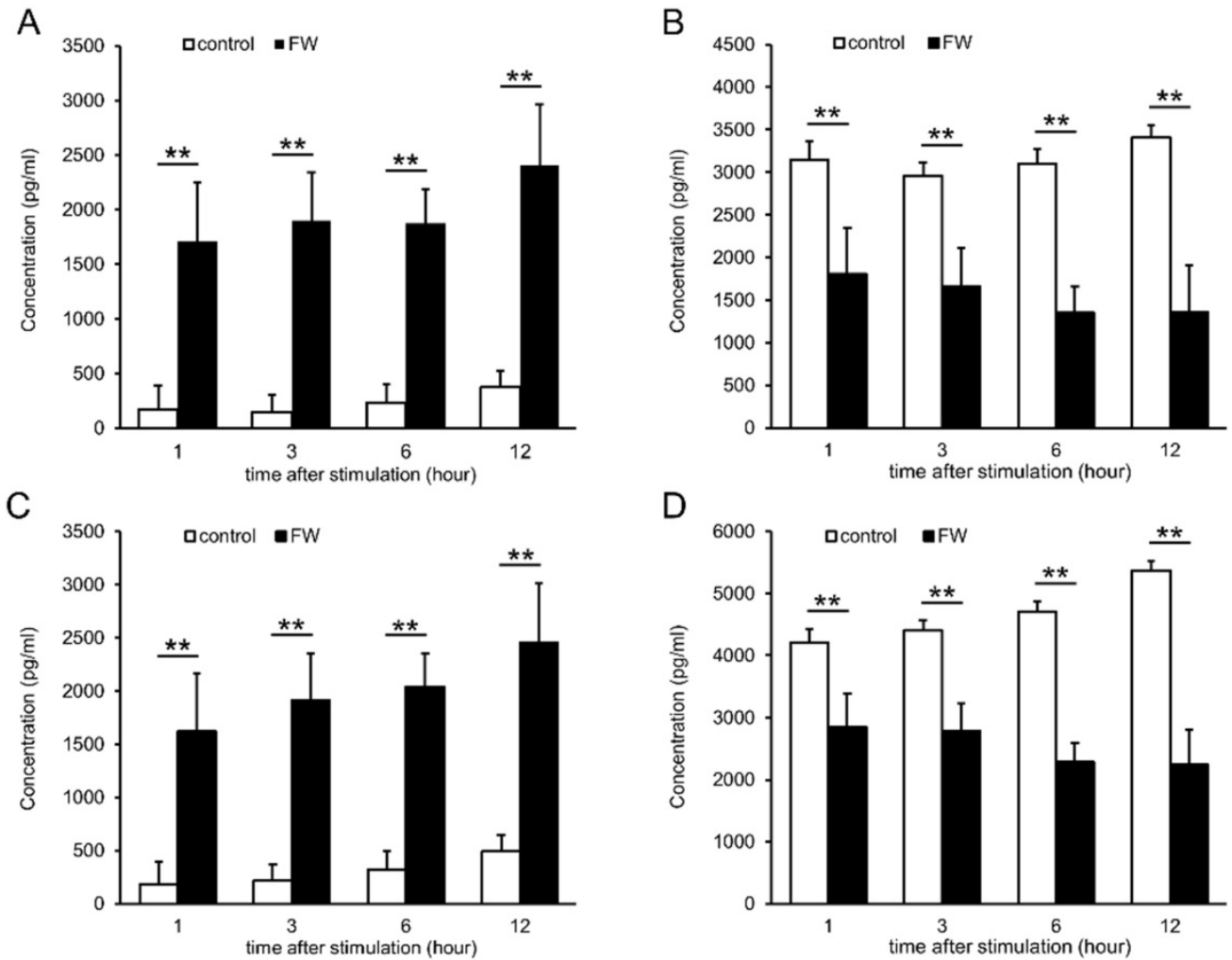

Figure 2. FW-treatment significantly augmented the secretion of EMMPRIN. HSC3 (A, B) and Ca9-22 (C, D) cells were treated with or without FW for 30 sec. After wash, the cells were further cultured for 1, 3, 6 and $12 \mathrm{~h}$. The culture supernatants and the cell lysates were harvested and subjected to ELISA. In both cell lines, EMMPRIN in the culture supernatants augmented drastically. In contrast, EMMPRIN in cell lysates dropped significantly. The mean \pm standard deviation (SD) of three independent experiments are shown. $\left({ }^{* *} \mathrm{P}<0.01\right)$

A

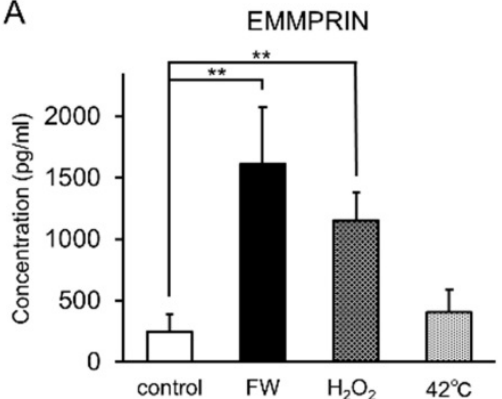

B

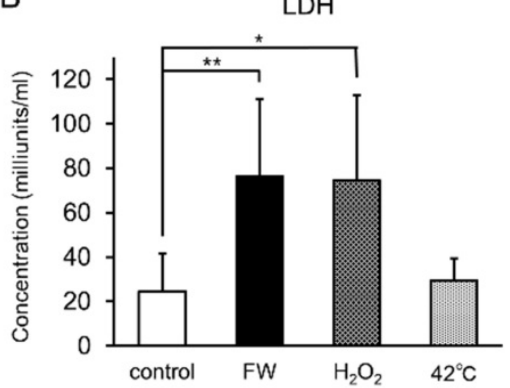

C

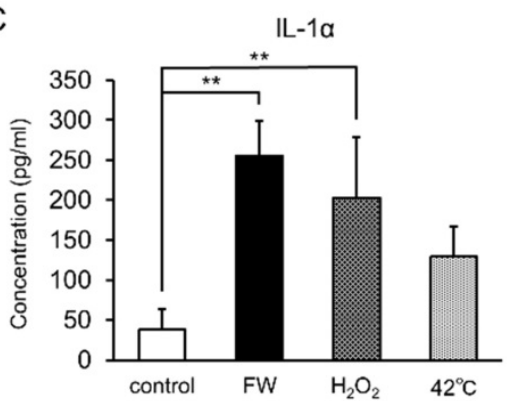

Figure 3. EMMPRIN secretion paralleled LDH release and IL- $1 \alpha$ secretion. $\mathrm{HSC} 3$ cells were cultured under stress conditions $\left(\mathrm{FW}\right.$-stimulated, $10 \mathrm{mM} \mathrm{H}_{2} \mathrm{O}_{2}$ treatment or cultured at $42^{\circ} \mathrm{C}$ ) for $3 \mathrm{~h}$. The culture supernatants were harvested and EMMPRIN (A), LDH (B) and IL-1a (C) concentrations were measured. FW-and 10 mM $\mathrm{H}_{2} \mathrm{O}_{2}$-treatment induced both EMMPRIN, LDH and IL-1 $\alpha$ secretion to the culture supernatnats. IL-1 $\alpha$ secretion was only slightly augmented at $42^{\circ} \mathrm{C}$. The mean \pm SD of three independent experiments are shown. $\left({ }^{*} \mathrm{P}<0.05,{ }^{* *} \mathrm{P}<0.01\right)$

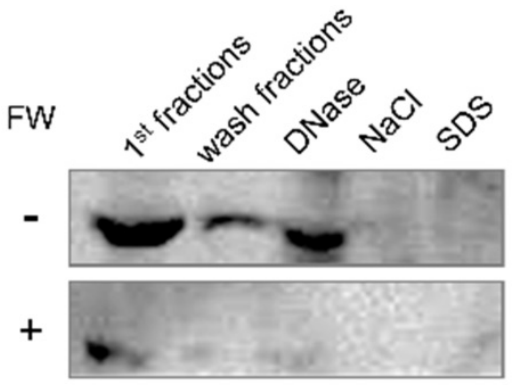

Figure 4. The intracellular localization of EMMPRIN before and after FW-treatment. HSC3 cells were treated with or without FW for $30 \mathrm{~s}$, and further cultured for $1 \mathrm{~h}$. The cells were collected and processed sequentially with Trion X-100 buffer twice (1 1 st and wash fractions), DNase buffer (DNase), $\mathrm{NaCl}$ buffer $(\mathrm{NaCl})$ and SDS buffer (SDS). The extracted samples were loaded on SDS-PAGE and subjected to Western blotting. EMMPRIN in the 1st fraction disappeared rapidly after FW-treatment. The representative of four independent experiments are shown.
Next, we examined the functionality of the released EMMPRIN. HSC3 cells were treated with or without FW and cultured for $3 \mathrm{~h}$. At the end of the culture, the supernatants (conditioned media) were collected. The human monocytic cell line, THP1 was cultured with these conditioned media and the expression of various EMMPRIN target genes were examined by real-time PCR. The FW-treated conditioned medium $(\mathrm{FW}(+))$ significantly induced the expression of PDGF, MMP1, MMP2, MMP8, MMP9, MMP13, MMP14 and IL-8 (Fig 5A); in contrast, only VEGF expression was reduced (Fig 5A). To further confirm EMMPRIN-mediated induction of its target genes, the EMMPRIN in the conditioned 


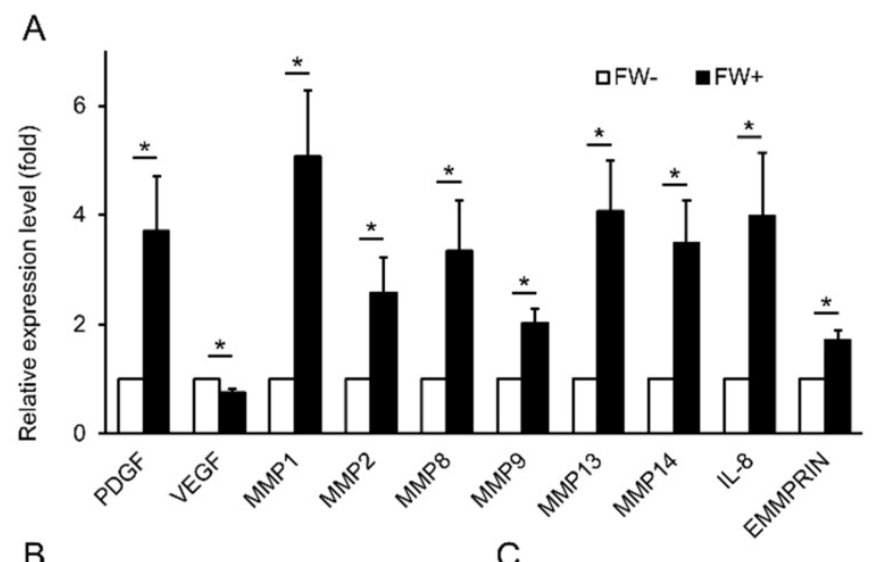

B

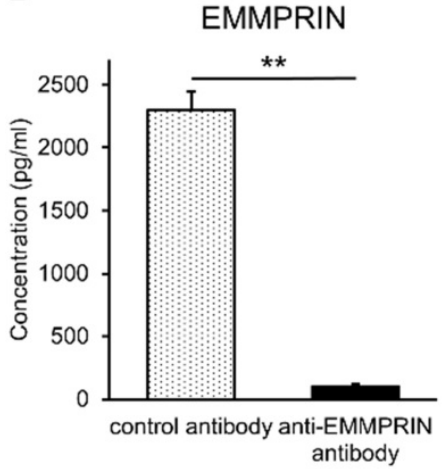

C

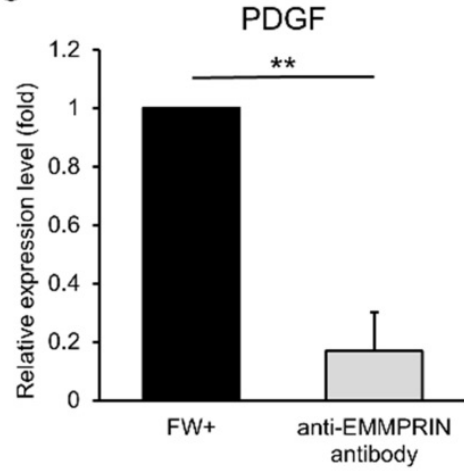

Figure 5. FW-induced EMMPRIN augmented the expression of its target genes in THPI cells. (A) HSC 3 cells were stimulated with or without FW for $30 \mathrm{~s}$ and further cultured for $3 \mathrm{~h}$. The culture supernatants from $\mathrm{FW}$-treated $(\mathrm{FW}+)$ or -untreated (FW-) were harvested. THPI cells were stimulated with the FW+ or FW- for $3 \mathrm{~h}$. At the end of culture, the total RNA was purified and subjected to real-time PCR. The basal expression levels of each target gene was set as 1 and relative expression level was shown as fold-induction. Most of the target genes, but VEGF, were augmented by FW+. (B) The anti-EMMPRIN antibody or control antibody was added to $\mathrm{FW}+$ and rotated for $18 \mathrm{~h}$ followed by protein G-sepharose to eliminate EMMPRIN from FW+. After immunoprecipitation, EMMPRIN concentration was measured by ELISA. Addition of anti-EMMPRIN antibody totally eliminated EMMPRIN from FW+. (C) THPI cells were stimulated with the immunoprecipitated (anti-EMMPRIN antibody) or control antibody-treated conditioned medium (FW+). Total RNA was purified and subjected to real-time PCR to examine PDGF expression. FW+ augmented the expression of PDGF and this value was set as 1 . In contrast, anti-EMMPRIN antibody-treated sample failed to augment PDGF expression in THP-1 cells. The mean \pm SD of at least three independent experiments are shown. $\left({ }^{*} \mathrm{P}<0.05,{ }^{* *} \mathrm{P}<0.01\right)$

medium was eliminated by immunoprecipitation. EMMPRIN concentration in Ab-treated FW (+) was significantly reduced to less than $100 \mathrm{pg} / \mathrm{ml}$ when compared with Ab-treated conditioned medium in the control $(2,300 \mathrm{pg} / \mathrm{ml}$; Fig 5B). Using these conditioned media, the THP1 cells were stimulated and PDGF mRNA expression was examined. The treatment of THP1 cells with Ab-treated FW (+) resulted in reduced PDGF levels (Fig 5C) indicating an EMMPRIN-mediated induction of PDGF in the THP1 cells.

\section{Discussion}

FW and $\mathrm{H}_{2} \mathrm{O}_{2}$ stimulation resulted in the augmented secretion of EMMPRIN in the culture medium. We examined the two-different cell lines, HSC3 and Ca9-22, to demonstrate that the FW-induced EMMPRIN secretion is a general phenomenon for oral squamous cell carcinoma cells.
This change was independent of the transcriptional regulation. Moreover, intracellular EMMPRIN levels were concomitantly reduced. These results clearly indicated that EMMPRIN was released from the intracellular storage in response to the stimuli. In order to examine the intracellular localization of EMMPRIN, cell fractionation experiments were performed. EMMPRIN is a transmembrane glycoprotein belonging to the immunoglobulin superfamily [4-6], and was thus expected to be localized exclusively on the cell surface. The $1^{\text {st }}$ and wash fractions contained cytoplasmically localized proteins, and most of the EMMPRIN was localized in these fractions. The triton-X100-based buffer is not strong enough to extract the transmembrane proteins; therefore, a part of the EMMPRIN might be attached to the cell membrane or localized in the cytoplasm by unknown mechanisms. A typical alarmin IL-1a may demonstrate membrane attachment; the precursor form of IL-1a bound to the plasma membrane via a lectin-like interaction and this interaction is dissociated by D-mannose treatment [14]. Interestingly, EMMPRIN can be detected in DNase-sensitive fractions. DNase destroys the basic genomic DNA structure and this fraction is expected to contain the proteins interacting with DNA. If this is the case, EMMPRIN might be localized in the nucleus. Although the implication of this finding remains elusive, the intracellular localization of EMMPRIN needs to be examined further. FW-treatment drastically reduced EMMPRIN in the all fractions in this study suggesting that the EMMPRIN secreted by FW stimulation is derived from cytoplasmic or nuclear storages.

EMMPRIN secretion paralleled LDH release in the cells, which were thought to present with a certain degree of damage. Various alarmins such as high mobility group box-1 protein (HMGB1) and IL-1a have been described to date [11]. The common features of these alarmins include the following: intranuclear localization, lack of signal peptides, ER-Golgi-independent secretion pathway, and other immunogenic functions.

Consistent with other alarmins rapid EMMPRIN secretion was noted following FW-treatment. Cell fractionation analysis suggested an intranuclear localization of the protein. The localization of other alarmins such as IL-1a and HMGB-1 is generally observed in the nucleus due to nuclear localizing signals (NSL) [15, 16]. Moreover, IL-1a has been 
reported to contribute to the transcriptional expression of its target genes [17]. On the other hand, EMMPRIN does not have NLS. The intranuclear localizing mechanisms and functions of EMMPRIN are unclear, and merit further studies.

The most important function of EMMPRIN is to induce the production of MMPs [4]. THP1 cells were stimulated with conditioned medium derived from the FW-treated or -untreated HSC3 cells in order to examine whether the EMMPRIN released after FW stimulation was functional. The conditioned medium successfully induced the expression of various MMPs, PDGF and IL-8. Although both EMMPRIN and PDGF contribute to the wound healing, no reports correlating these two factors have been observed. For these reasons, we concentrated on the EMMPRIN-induced PDGF up-regulation. Moreover, the enhancing effect was abolished when the EMMPRIN in the conditioned medium was removed by immunoprecipitation. These results indicated that EMMPRIN exerted its effects on the monocytic cells. The monocyte is one of the most important immune cells in the body. EMMPRIN was able to activate the THP1 cells in the present study, thus fulfilling the fourth criteria of alarmins.

Two different kinds of EMMPRIN receptors have been described, the receptor for advanced glycation end product (RAGE) and toll-like receptor 4 (TLR4) [3]. The expressions of both receptors in THP1 cells were confirmed by reverse transcriptase-PCR (data not shown), and EMMPRIN signal should be transduced by these receptors. Among the genes analyzed, only VEGF was down regulated by EMMPRIN. This observation was inconsistent with previous reports showing EMMPRIN-mediated VEGF induction [18, 19]. Another recent study further demonstrated EMMPRIN-mediated bone-marrowderived cell recruitment to the cancer region [20]. Although the reasons for the discrepancy between the findings are unknown, it may be noteworthy that the previous studies used lung cancer and endothelial cells. EMMPRIN might exert various effects in a cell type-specific manner. Thus, the downstream signaling pathways leading to MMPs induction or VEGF reduction might be different between cells, and will need to be clarified in more detail in future.

The expression levels of EMMPRIN are strongly associated with the prognosis of cancer [21]. In the present study, FW was shown to induce the secretion of EMMPRIN from the cellular storages of the oral cancer cells. As FW is useful for disinfection and the acceleration of wound healing, it is indispensable to know whether FW-treatment can augment EMMPRIN secretion from normal cells such as fibroblasts or epithelial cells. The results obtained in this study suggested that its clinical use in the field of cancer warrants careful attention.

\section{Supplementary Material}

Supplementary figure.

http://www.medsci.org/v15p1365s1.pdf

\section{Acknowledgements}

This study was supported in part by research grants from Sato Fund from the Nihon University School of Dentistry and a grant from the Dental Research Center, Nihon University School of Dentistry, a Nihon University multidisciplinary research grant and Individual Research Grant and MEXT-Supported Program for the Strategic Research Foundation at Private Universities 2013-2017. Grant-in-Aid for Scientific Research (C) (MEXT).

\section{Competing Interests}

The authors have declared that no competing interest exists.

\section{References}

1. Kessenbrock K, Plaks V, Werb Z. Matrix metalloproteinases: regulators of the tumor microenvironment. Cell. 2010; 141: 52-67.

2. Basset P, Wolf C, Chambon P. Expression of the stromelysin-3 gene in fibroblastic cells of invasive carcinomas of the breast and other human tissues: a review. Breast Cancer Res Treat. 1993; 24: 185-193.

3. Iacono KT, Brown AL, Greene MI et al. CD147 immunoglobulin superfamily receptor function and role in pathology. Exp Mol Pathol. 2007; 83: 283-295.

4. Fossum S, Mallett S, Barclay AN. The MRC OX-47 antigen is a member of the immunoglobulin superfamily with an unusual transmembrane sequence. Eur J Immunol. 1991; 21: 671-679.

5. Miyauchi T, Masuzawa Y, Muramatsu T. The basigin group of the immunoglobulin superfamily: complete conservation of a segment in and around transmembrane domains of human and mouse basigin and chicken HT7 antigen. J Biochem. 1991; 110: 770-774.

6. Biswas $\mathrm{C}$, Zhang $\mathrm{Y}$, DeCastro $\mathrm{R}$ et al. The human tumor cell-derived collagenase stimulatory factor (renamed EMMPRIN) is a member of the immunoglobulin superfamily. Cancer Res. 1995; 55: 434-439.

7. Muramatsu T, Miyauchi T. Basigin (CD147): a multifunctional transmembrane protein involved in reproduction, neural function, inflammation and tumor invasion. Histol Histopathol. 2003; 18: 981-987.

8. Sun J, Hemler ME. Regulation of MMP-1 and MMP-2 production through CD147/extracellular matrix metalloproteinase inducer interactions. Cancer Res. 2001; 61: 2276-2281.

9. Kubota A, Goda T, Tsuru T et al. Efficacy and safety of strong acid electrolyzed water for peritoneal lavage to prevent surgical site infection in patients with perforated appendicitis. Surg Today. 2015; 45: 876-879.

10. Saiki A, Motoyoshi M, Motozawa K et al. EMMPRIN inhibits bFGF-induced IL-6 secretion in an osteoblastic cell line, MC3T3-E1. Int J Med Sci. 2017; 14: $1173-1180$

11. Oppenheim JJ, Yang D. Alarmins: chemotactic activators of immune responses. Curr Opin Immunol. 2005; 17: 359-365.

12. Omagari D, Mikami Y, Suguro H et al. Poly I: C-induced expression of intercellular adhesion molecule-1 in intestinal epithelial cells. Clin Exp Immunol. 2009; 156: 294-302.

13. Furuta M, Kose S, Koike M et al. Heat-shock induced nuclear retention and recycling inhibition of importin a. Genes Cells. 2004; 9: 429-41.

14. Brody DT, Durum SK. Membrane IL-1: IL-1 alpha precursor binds to the plasma membrane via a lectin-like interaction. J Immunol. 1989; 143: 1183-1187.

15. Wesndorf JH, Garfinkel S, Zhan X et al. Identification of a nuclear localization sequence within the structure of the human interleukin-1 alpha precursor. J Biol Chem. 1993; 268: 22100-22104.

16. Maier JA, Statuto M, Ragnotti G. Endogenous interleukin 1 alpha must be transported to the nucleus to exert its activity in human endothelial cells. Mol Cell Biol. 1994; 14: 1845-1851.

17. Werman A, Werman-Venkert $R$, White $R$ et al. The precursor form of IL-1a is an intracrine proinflammatory activator of transcription. Proc Natl Acad Sci U S A. 2004; 101: 2434-2439. 
18. Tang Y, Nakada MT, Rafferty P et al. Regulation of vascular endothelial growth factor expression by EMMPRIN via the PI3K-Akt signaling pathway. Mol Cancer Res. 2006; 4: 371-377.

19. Bougatef $\mathrm{F}$, Quemener $\mathrm{C}$, Kellouche $\mathrm{S}$ et al. EMMPRIN promotes angiogenesis through hypoxia-inducible factor-2alpha-mediated regulation of soluble VEGF isoforms and their receptor VEGFR-2. Blood. 2009; 114: 5547-5556.

20. Chen $Y$, Gou X, Kong DK et al. EMMPRIN regulates tumor growth and metastasis by recruiting bone marrow-derived cells through paracrine signaling of SDF-1 and VEGF. Oncotarget. 2015; 6: 32575-32585.

21. Weidle UH, Scheuer W, Eggle D et al. Cancer-related issues of CD147. Cancer Genomics Proteomics. 2010; 7: 157-169. 